\title{
Clinical Study of Hashimotos Thyroiditis
}

\author{
Dr B Sri Lekha ${ }^{1}$,Dr G Raghuveer Chakravarthy ${ }^{2}$,Dr K.A.S.S.N.Kalyan ${ }^{3}$ \\ ${ }^{I}$ Dr B Sri Lekha, Student, Dr.Pinnamaneni Siddartha Institute Of Medical Sciences \& Research Foundation, \\ Chinaoutapalli, Krishna Dist, Andhra Pradesh, India. \\ ${ }^{2}$ Dr. G Raghuveer Chakravarthy, Assistant Professor, General Surgery, Dr.Pinnamaneni Siddartha Institute Of \\ Medical Sciences \& Research Foundation, Chinaoutapalli, Krishna Dist, Andhra Pradesh, India. \\ ${ }^{3}$ Dr. K.A.S.S.N.Kalyan ,Junior Resident, General Surgery, Dr.Pinnamaneni Siddhartha Institute Of Medical \\ Sciences \& Research Foundation, Chinaoutapalli, Krishna Dist, Andhra Pradesh, India.
}

\begin{abstract}
Background And Objective

Hashimoto's thyroidits is a common cause of goitrous enlargement with hypothyroidism. This study was done to study various clinical presentation with thyroid function and to diagnose and treat the cases with thyroxine and how manycases wanted surgical procedure.

METHODS: Patients presenting to surgical out patient with goitrous enlargement were subjected to hormonal assay and FNAC. If FNAC showed features of lymhocytic infiltration they were subjected to autoantibody titers. All the patients with Hashimoto's thyroidits were put on Thyroxine and regular fllow-up was done to note the clinical signs and symptoms including the size of the goiter. Patients with obstructive symptoms or goiter in whom size did not reduce or for cosmetic reasons were operated.

Results: Hashimoto's thyroidits was more in females and maximum in 30-40 years of age group. Out of the 93 cases with the history from 1 month to 4 years, $62 \%$ were multinodular goiter, 35\% were diffuse goiter and $2.5 \%$ was solitary nodule. Regarding thyroid function $47 \%$ were hypothyroid, $35 \%$ were Euthyroid and 17\%were hyperthyroid. FNAC showed Hashimoto's thyroiditis in about 86 patients out of 93 cases studied. Antibody titers were positive for $87.5 \%$ of cases, 11 cases were operated out of which 4 cases had obstructive symptoms.

Interpretation And Conclusion: Hashimoto's thyroidits is more common in females, maximum between $30-40$ years. Most of them had multinodular and diffuse goiters and were in hypothyroid state. Most of the cases were diagnosed with FNAC and treated medically with thyroxine replacement and surgery was rarely required.
\end{abstract}

Keywords: FNAC-Fine needle aspiration cytology, Goitre, Hashimotos, Multinodular

\section{Introduction}

Hashimoto's thyroiditis, a well known condition affecting the thyroid gland is often under diagnosed due to its wide spectrum of clinical manifestations. As such it could present as a multinodular goitre, a diffuse goitre or a solitary nodule with features of hypothyroidism, hyperthyroidism or in a euthyroid state. It is the commonest cause of goitre with hypothyroidism in iodine sufficient areas. Since Hakaru Hashimoto discovered chronic lymphocytic infiltration in the thyroid in 1912, a sea change has occurred in our knowledge of this autoimmune process. It is now well documented that not all cases with histological features of lymphocytic infiltration are due to Hashimoto's thyroiditis. This histological picture of lymphocytic infiltration is also seen in some other conditions affecting the thyroid. The bio-chemical evaluation of autoantibody titers (AMA, ATG) together with

the clinical, FNAC and hormone assay guides the clinician towards making a diagnosis of Hashimoto's thyroiditis. This condition is managed conservatively and surgery is rarely required.

\section{Objectives}

1. To detect cases of Hashimoto's thyroiditis by FNAC, positive antibody titres or final histopathology.

2. To study the varied clinical pattern of these cases.

3. To detect the number of cases of Hashimoto's thyroiditis that present with biochemical features of hypo/hyperthyroidism / Euthyroid state.

4. Correlation of FNAC and hormone assay with autoantibodies against the thyroidgland.

5. To determine the number of cases of Hashimoto's thyroiditis that are actuallytaken up for surgery.

\section{Materials And Methods}

93 cases of Hashimoto's thyroiditis were studied from Aug 2012 to July 2015. Data was collected from 93 patients, both outpatients and inpatients in this period, treated at Tertiary Care teaching Hospital. 
Patients were informed about the study, the relevance of the investigations, the "non operable" treatment modality, the requirement of daily thyroxine supplements presumably for an indefinite period of time and the need for regular follow up.

All the investigations required for the study were usually done on outpatient basis. Patient who underwent surgery was investigated during their admission period prior to surgery. All patients presenting to the surgical outpatient with goitrous enlargement were subjected to a hormonal assay and FNAC. If FNAC showed features of lymphocytic infiltration, then thyroid autoantibody estimation were ordered for. However, if FNAC showed no features of lymphocytic infiltration, but the patient was in subclinical or overt hypothyroidism, even then antibody estimation were done. The patient who underwent surgery, had an additional spectra of preoperative investigations with routine blood, urine, chest and neck X-rays, ECG and an indirect laryngoscope examination. They were duly certified "fit" by the attending physicians and went on to undergo subtotal thyroidectomies. Post-operative histopathological

Examinations of the thyroid specimens were done in all cases. Immense care was taken in obtaining a detailed history and meticulous local and systemic examinations were done. The data thus obtained was recorded on a proforma prepared exclusively for the study.TT3,TT4,TSH \& TPO antibodies were analysed by Lumax 4100 (Chemiluminescence) and tabulated Fasting samples were taken

\section{Inclusion Criteria}

All patients diagnosed as Hashimoto's thyroiditis were included. Diagnosing could be on the basis of FNAC, positive antibody status or final histopathology report.

\section{Exclusion Criteria}

All patients diagnosed to have thyroid malignancy prior to their admission to Tertiary Care Hospital

\section{Observations}

During the study period 93 cases of Hashimoto's thyroiditis were treated. Out of which 90 were females and 3 male patients. These patients were studied with history, clinical sign and symptoms and investigated and recorded in the proforma and the following observations were made and compared with other studies.

In this study of 93 cases of Hashimoto's thyroiditis, the following observations were made:

\section{Sex Distribution}

Table 1:Showing sex distribution

\begin{tabular}{|l|l|l|}
\hline No. of cases & Males & Females \\
\hline 93 & & \\
\hline & 3 & 90 \\
\hline
\end{tabular}

\section{Age Distribution}

The age distribution of cases in our study is as follows table 2:

\begin{tabular}{|l|l|l|}
\hline Age (in years) & Female & Male \\
\hline & & \\
\hline$<20$ & 5 & - \\
\hline & & \\
\hline $20-29$ & 28 & - \\
\hline & & \\
\hline $30-39$ & 29 & - \\
\hline & & \\
\hline $40-49$ & 24 & 1 \\
\hline & & \\
\hline$>50$ & 4 & 2 \\
\hline & & $\mathbf{3}$ \\
\hline Total & $\mathbf{9 0}$ & \\
\hline & &
\end{tabular}

The age of the patients in this study ranged from 12 - 55 years. The youngest being a 12 year old age girl and the oldest being a 55 years old woman. Most cases were in the twenty to thirty age groupsThe average age of the patient in the age study being 33.46 years. 


\section{Mode Of Presentation}

All 93 patients presented with history of swelling in front of the neck. 16 patients has features of hyperthyroidism and 4 patients presented with the obstructive symptoms of dysphagia.

Table 3 showing mode of presentation:

\begin{tabular}{|l|l|}
\hline SYMPTOMS & NO. OF PATIENTS \\
\hline Swelling & 93 \\
\hline & \\
\hline Toxic symptoms & 16 \\
\hline & \\
\hline Obstructive symptoms & 4 \\
\hline Pain & \\
\hline & Nil \\
\hline Change in Voice & \\
\hline & Nil \\
\hline & \\
\hline
\end{tabular}

\section{Clinical Presentations Of The Swelling}

Table 4 showing clinical presentation: Table:

\begin{tabular}{|l|l|l|}
\hline Clinical Presentation & No. of Patients & Percentage \\
\hline MNG & & \\
\hline & 58 & $62.50 \%$ \\
\hline Diffuse enlargement & & \\
\hline & 33 & $35 \%$ \\
\hline Solitary Nodule & & \\
\hline & 2 & $2.5 \%$ \\
\hline
\end{tabular}

Out of these 93 cases. 58 cases presented as multi nodular goiter, 33 cases as diffuse enlargement and 2 cases presented as solitary nodule.

\section{Duration Of Swelling}

Table 5 showing duration of swelling:

\begin{tabular}{|c|c|c|}
\hline \multicolumn{2}{|c|}{ Duration Of } & Number Of \\
\hline \multicolumn{2}{|c|}{ Swelling } & Patients \\
\hline \multicolumn{2}{|c|}{$0-6$ Months } & 47 \\
\hline \multicolumn{2}{|c|}{6 months -1 Year } & 27 \\
\hline 1 & -2 Years & 15 \\
\hline 2 & -3 Years & 4 \\
\hline & $>3$ Years & 2 \\
\hline
\end{tabular}

The duration of swellings ranged from 1 month to 4 years. Maximum number of cases presented within six months of noticing theswelling.

\section{Thyroid Status At Presentation}

Thyroid profile for estimation of $\mathrm{TSH}, \mathrm{TT}_{3}$ and $\mathrm{TT}_{4}$ and $\mathrm{TPO}$ antibodies were done in all 93 patients. The results are as follows:

Out of the 93 cases 44 patients had hypothyroidism, 16 cases had hyperthyroidism and 33 patients were euthyroid.

Thyroid status at presentation: table 6

\begin{tabular}{|l|l|l|}
\hline Thyroid Status & No. of Patients & Percentage \\
\hline Hypothyroid & 44 & $47.50 \%$ \\
\hline Euthyroid & 33 & $35 \%$ \\
\hline Hyperthyroid & 16 & $17.5 \%$ \\
\hline
\end{tabular}




\section{Fnac Status At Presentation}

All 93 patients underwent FNAC and in 86 of them a cytopathological diagnosis of Hashimoto's thyroiditis was made and 7 cases were reported as colloid goitre.

Table 7 showing FNAC reports at presentation

\begin{tabular}{|l|l|l|}
\hline FNAC & Number of patients & Percentage \\
\hline Hashimoto's & 86 & $92.5 \%$ \\
\hline Thyroiditis & & \\
\hline colloid goitre. & 7 & $7.5 \%$ \\
\hline
\end{tabular}

\section{Antibodies At Presentation}

All 93 patients had their thyroid autoantibody estimation done.Antibody estimations were done by Chemiluminescence method using Lumax 4100.

Antibodies at presentation: table 8

\begin{tabular}{|l|l|l|}
\hline Antibodies & number of & Percentage \\
\hline estimation & patients & \\
\hline Both Antibodies Positive & 47 & 50 \\
\hline AMA and ATG & & \\
\hline Both Antibodies Negative & 12 & $12.50 \%$ \\
\hline AMA and ATG & & \\
\hline Only AMA Positive & 28 & 30.00 \\
\hline Only ATG Positive & 6 & $7.50 \%$ \\
\hline
\end{tabular}

\section{Clinical And Laboratory Findings}

Table showing patients clinical presentation and laboratory findings:

Table: 9

\begin{tabular}{|l|l|l|l|}
\hline Clinical / Laboratory & Euthyroid & Hypothyroid & Hyperthyroid \\
\hline findings & & & \\
\hline No. of Cases & 33 & 44 & 16 \\
\hline Diffuse goiter & 12 & 19 & 2 \\
\hline Multinodular goiter & 19 & 25 & 14 \\
\hline Solitary nodular goiter & 2 & - & - \\
\hline Both antibodies positive & 14 & 21 & 12 \\
\hline Both antibodies negative & - & 12 & - \\
\hline Only AMA positive & 14 & 10 & 4 \\
\hline Only ATG positive & 4 & 2 & - \\
\hline
\end{tabular}

Surgery

All but 11 cases were treated with suppression dose of thyroxine and in all cases the goiter reduced in size and patients remained symptom free.

Totally for 11 cases, subtotal thyroidectomy was done. Out of these, 6 cases FNAC was colloid goitre and histopathology report was Hashimoto's Thyroiditis and other 5 cases were operated for the swelling which did not reduce in size and for cosmetic reason and for obstructive symptoms. thyroxine.

None of them had any post operative complications, all the 11 cases were put on replacement

\section{Discussion}

During the study period from Aug 2012 to July 2015, 93 patients were detected to have Hashimoto's thyroiditis either by FNAC, antibody titers or final histopathology reports.

Female preponderance is a well established feature of thyroid diseases and our study was no different having a strong female preponderance with 90 females and 3 male patient, the male to female ratio being $1: 30$. This coincides with the observations made by Sharma et al ${ }^{1}$, Joseph et al ${ }^{2}$, and Fenn et al ${ }^{3}$, all of whom observed a female preponderance, with a male to female ratio of 1: 13, 1: 12 and 1; 14 respectively. Though ratio is higher in our study, this difference in ratio of male to female was because of smaller sample in our study. 
In our study the age incidence ranged from 12 years to 55 years, the youngest being a 12 years old girl and oldest being a 55 year old woman. The average age in this study was 33.45 years and highest incidence being in the $30-39$ year age group. Lakshmana Rao et al ${ }^{4}$, had an average age of 40.4 years in their study and Fenn et al $1^{3}$ observed that most of the patients were in their $4^{\text {th }}$ to $5^{\text {th }}$ decade of life.

All the patients presented with a history of swelling in front of the neck, 7 patients with additional features suggestive of hyperthyroidism and 4 patients presenting with the additional features of obstruction. All the goiters were were firm in consistency.

In this study, the majority of the cases were Multinodular goiters $62.5 \%, 35 \%$ being diffuse goiters and $2.5 \%$ being solitary nodules. Similarly Rao. $\mathrm{KS}^{5}$, in his series reported a predominance of multinodular goiters and $10.53 \%$ being solitary nodule. However, Kusum Kapila et al ${ }^{6}$, in their observations had a preponderance of diffuse goiters with $55.80 \%$, multinodular goiters and solitary nodules amounting for $18.30 \%$ and $25.90 \%$ respectively.

The hypothyroid and euthyroid patients in our study accounted for $47.5 \%$ and $35 \%$ respectively, while the hyperthyroid patients accounted for $17.5 \%$ of the cases. In the studies of Fenn et al ${ }^{3}$ and Kusum Kapila et al 6 , the euthyroid patients amounted for $46.66 \%$ and $79.30 \%$; the hypothyroid patients for $44.44 \%$ and $10.10 \%$, and the hyperthyroid patients for $6.66 \%$ and $6.90 \%$ respectively.

In our study $15.6 \%$ of the patients were in subclinical hypothyroidism, whereas in the study of Marhawa et al $^{7} 18.60 \%$ of the patients were in subclinical hypothyroidism. In this study FNAC was positive in $92.5 \%$ of the cases, in sharp contrast to the observation made by Lakshmana Rao et al ${ }^{8}$ who with a 14-16 gauge needle achieved an accuracy of $77.70 \%$. In our series $87.5 \%$ of the cases had thyroid autoantibodies positive. The series of Hasanat et $\mathrm{al}^{9}$ and Lakshamana Rao et $\mathrm{al}^{8}$ reported thyroid auto antibodies positivity of $63 \%$ and $83.34 \%$ respectively. In our study, in the group that was hypothyroid at presentation, thyroid autoanitibodies were positive in $66.6 \%$ of cases. In the euthyroid and hyperthyroid groups the thyroid antibody positivity was $100 \%$. Hasanat et al ${ }^{9}$ reported thyroid autoantibody positivity of $55 \%$ and $37 \%$ in their hypothyroid and hyperthyroid groups respectively.

The patients were followed up regularly at intervals of 3 months, and at every visit the pulse, weight, consistency of the gland and diameter of the neck were recorded.

All the hypothyroid and euthyroid patients were put on thyroxine replacement and thyroxine suppression therapy respectively and monitored clinically. Eventually all the patients were found to be clinically euthyroid.

All the patients with diffuse goiters and solitary nodules put on hormonal therapy with thyroxine showed a decrease in size of the gland that was appreciated by manual palpation and by measuring the girth of the neck. The patients with multinodular goiters showed no increase or decrease in size of the gland.

11 patients underwent surgery, of these 6 were colloid goiters and underwent subtotal thyroidectomies, later on diagnosed as Hashimoto's thyroiditis by histopathology. Surgery done for the other 5 patients was because of cosmetic purpose and obstructive symptoms.

\section{Summary}

93 patients were diagnosed to have Hashimoto's thyroiditis in the study period from May 2013 to June 2015. The study was conducted at Tertiary Care Teaching Hospital A brief introduction has been presented with detailed discussion The findings of our study were compared with that of the available literature.

\section{The findings of our study are as follows:}

1. The occurrence of Hashimoto's thyroiditis was maximum in the $30-34$ year age group.

2. Females outnumbered the males with a male to female ratio of $1: 30$.

3. All the 93 patients presented with complaints of swelling in front of the neck. 4 patients presented with obstructive symptoms as well.

4. Duration of the swelling ranged from 1 month to 4 years, however, most of the patients presented within 6 months of noticing the swelling.

5. Consistency of the gland was firm in all cases.

6. In our study, 58 patients were multinodular goiter (62.5\%), 33 were diffuse goiters (35\%) and 2 was solitary nodule $(2.5 \%)$.

7. 44 patients were hypothyroid (47.5\%), 33 patients were Euthyroid (35\%) and 16 patients were hyperthyroid $(17.5 \%)$.

8. FNAC was positive in 86 patients $(92.5 \%)$.

9. Antibodies were positive in 81 patients $(87.5 \%)$.

10. 82 patients were treated conservatively and monitored regularly every 3 months. Eventually, at the time of writing this article, all 82 patients were clinically euthyroid.

All diffuse goiters and solitary nodules regressed in size with thyroxine therapy. The multinodular goiters 
showed no increase or decrease in size of the gland.

11. All but 11 patients were treated conservatively. 11 patients underwent surgery, 5 for cosmetic purpose and obstructive symptoms, other 6 cases underwent subtotal thyroidectomy for colloid goiters and histopathology revealed to be Hashimoto's thyroiditis.

\section{Conclusion}

Hashimoto's thyroiditis is a common cause of goitrous enlargement with hypothyroidism. Females are more prone to develop Hashimoto's thyroiditis. Hashimoto's thyroiditis has a varied clinical presentation and as such could present as a diffuse goiter, a multinodular goitre or a solitary nodule. Hashimoto's thyroiditis could present in a hypothyroid state, an euthyroid state, and in a small proportion of the patients in a hyperthyroid state.Diagnosis of Hashimoto's thyroiditis could be done by FNAC, positive antibody titers or final histopathology. Treatment is primarily medical with thyroxine replacement or suppression therapy and surgery is rarely required.Diffuse goiters and solitary nodules respond better to the medical line of management, than do multinodular goiters.

\section{References}

[1]. Sharma AK, Paliwal RK, and Pendse AK. "Hashimoto's Thyroiditis - Clinical Review”. Journal of Post Graduate Medicine, 1990; $36(2): 87-90$

[2]. Joesph TM and Joseph LBM. “Thyroiditis”. Indian Journal Of Surgery, 1967; 29: 293 -307.

[3]. Fenn AS, Job Ck, and Elizabeth George. "Hashimoto's thyroiditis”, Indian Journal of Surgery, 1980; 4: 123-125.

[4]. Lakshmana Rao K. M. and Reddy S. S . “ Hashimoto's disease - A Clinicopathological Study”. Indian Journal Of Surgery, December $1983 ;$ ( 45) : $693-695$.

[5]. Rao.K.S. "Hashimoto's Disease ( A Clinical - Pathological Study)”. The Clinician, May 1978; Volume 42, (5): 184 - 192.

[6]. Kusum Kapila, Sitara Adbul Sathar, Nawal Abdul Rahman Al - Rabah, Arun Prahash JC, and Man dalam S. Seshadri. "Chronic Lymhocytic (Hashimoto's) Thyroiditis in Kuwait Diagnosed by Fine Needle Aspirates". Ann. Saudi Med, 1995; 15 (4).

[7]. Marwaha RK, Garg MK, Nijhavan VS, Dham DN, Dubey R, Amberdar V, Jain SK and Mahendra NS. "Prevalence of Chronic Lymphocytic Thyroiditis in Adolescent Girls". Journal Of Association of Physicians of India, July 1998, (46), 606 - 608.

[8]. Lakshmana Rao K.M. and Reddy S. S. “ Hashimoto's disease - A clinicopathological Study”. Indian Journal Of Surgery, 1991; $\mathbf{5 3}(8-9), 338-342$

[9]. Hasabat MA, Rumi MAK, Alam MN, Nadim Hasan K, Salimullah M, Salam MA, Fariduddin M, Hajera Mahtab and Azad Dhan AK. "Status of antithyroid antibodies in Bangladesh". Postgraduate Medical Journal; 76 (896) : 345. 